\title{
Rendimiento pronóstico de reglas de decisión clínica en síncope Un estudio piloto
}

\section{Prognostic performance of clinical decision rules in syncope \\ A pilot study}

\author{
Arnold Méndez, Ingrid Tatiana Rojas, Óscar Ernesto Amarís, \\ Guillermo Mora • Bogotá, D.C. (Colombia)
}

\section{Resumen}

Objetivo: desarrollar un estudio piloto de factibilidad de comparación del rendimiento pronóstico de las reglas de decisión clínica en pacientes con síncope. Objetivo secundario: evaluar el rendimiento pronóstico de las reglas de decisión OESIL (Osservatorio Epidemiologico sulla Síncope nel Lazio), EGSYS (European Guidelines in Syncope Study), SFSR (San Francisco Syncope Rule) y su aplicación con los Ottawa Electrocardiographic Criteria (SFSR+Ottawa), en predicción de mortalidad por cualquier causa y desenlaces cardiovasculares y no cardiovasculares mayores a siete y 30 días.

Métodos: estudio observacional, analítico, prospectivo, tipo longitudinal, de no intervención, con muestreo por conveniencia. Se incluyeron pacientes $>18$ años de edad admitidos a urgencias con síncope menor o igual a 48 horas de evolución. Se compararon las reglas mediante curvas ROC y sensibilidad, especificidad, VPP y VPN para mortalidad y desenlaces mayores a siete y 30 días.

Resultados: se incluyeron 44 pacientes durante un periodo de siete meses, hospitalizándose el 100\%. El análisis ROC mostró una AUC para SFSR+Ottawa para mortalidad y/o desenlaces mayores a siete días de 0.76 (IC 95\% 0.49-0.82) y 30 días 0.76 (IC 95\% 0.49-0.82), con sensibilidad de 86 y $84 \%$ y especificidad de 45 y $47 \%$ a siete y 30 días respectivamente.

Conclusiones: es factible realizar un estudio de comparación de rendimiento pronóstico de reglas de decisión clínica de síncope en Colombia. La comparación realizada, sugiere un mejor desempeño de SFSR si se aplica con los criterios electrocardiográficos de Ottawa (SFSR+Ottawa) para la predicción de desenlaces a corto plazo. (Acta Med Colomb 2015; 40: 36-44).

Términos clave: síncope, reglas de predicción clínica.

\section{Abstract}

Objective: to develop a feasibility pilot study comparing the prognostic performance of clinical decision rules in patients with syncope. Secondary objective: To assess the prognostic performance of OESIL (Osservatorio sulla Epidemiologico Sincope nel Lazio) decision rules, EGSYS (European Guidelines in Syncope Study), SFSR (San Francisco Syncope Rule) and its application to the Ottawa Electrocardiographic Criteria (SFSR + Ottawa ) in predicting all-cause mortality and cardiovascular and non-cardiovascular outcomes longer than seven and 30 days.

Methods: an observational, analytical, prospective, longitudinal, non-interventional study with convenience sampling. Patients $>18$ years of age admitted to the emergency room less than or equal to 48 hours after onset of syncope were included. Rules were compared by using ROC curves and sensitivity, specificity, PPV and NPV for mortality and major outcomes to seven and 30 days.

Results: 44 patients were included over a period of seven months. $100 \%$ were hospitalized. ROC analysis showed an AUC for SFSR + Ottawa for mortality and / or major outcomes to 7 days of 0.76 (95\% CI 0.49 to 0.82 ) and 30 days 0.76 (95\% CI $0.49-0.82$ ) with sensitivity of 86 and $84 \%$ and specificity of 45 and $47 \%$ for seven and 30 days respectively.

Dr. Arnold Méndez Toro: Especialista en Medicina Interna, Servicio de Cuidado Intensivo, Fundación Hospital San Carlos; Dra. Ingrid Tatiana Rojas Ruiz: Enfermera, Especialista en Auditoría y Calidad y en Epidemiología; Dr. Óscar Ernesto Amarís Peña: Especialista en Medicina Interna y Cardiología, Profesor Asociado, Departamento de Medicina Interna, Facultad de Medicina, Universidad Nacional de Colombia; Dr. Guillermo Mora Pabón: Especialista Medicina Interna y Cardiología, Profesor Asociado, Facultad de Medicina, Universidad Nacional de Colombia. Bogotá, D.C. (Colombia).

Correspondencia. Dr. Guillermo Mora Pabón, Bogotá, D.C. (Colombia).

E-mail: gmorap@unal.edu.co

Recibido: 31/III/2014 Aceptado: 15/X/2014 
Conclusions: it is feasible to conduct a comparison study of prognostic performance of clinical decision rules of syncope in Colombia. The comparison realized suggests a better performance of SFSR if applied with electrocardiographic criteria of Ottawa (SFSR + Ottawa) for predicting outcomes in the short term. (Acta Med Colomb 2015; 40: 36-44).

Keywords: syncope, clinical prediction rules.

\section{Introducción}

El síncope como síndrome agrupa un conjunto de condiciones clínicas heterogéneas con implicaciones diagnósticas y terapéuticas en todos los matices de gravedad, lo que históricamente ha dificultado su aproximación (1).

No es fácil decidir entre una posible hospitalización innecesaria y el alta por la incertidumbre de encontrarse ante un riesgo de muerte alto en los días siguientes por una causa inadvertida. Por ello sería necesario contar con herramientas que permitan hacer un adecuado diagnóstico de las diferentes etiologías. Sin embargo, en la práctica clínica puede resultar complejo lograr un acertado diagnóstico e incluso decidir si optar por un manejo ambulatorio u hospitalario, por lo que se ha recurrido al desarrollo de reglas de decisión clínica que permitan medir el riesgo de mortalidad y desenlaces mayores y aproximarse a alguno de los grupos etiológicos. Sin embargo, a la fecha ninguno de estos modelos predictivos permite con certeza definir el riesgo vital a corto, mediano o largo plazo, ni confirma o descarta la causalidad. No se cuenta con evidencia incontrovertible que permita tomar una sola herramienta como la ideal o considerar que el uso de las reglas de decisión en esta patología supere al manejo convencional y al criterio médico (2).

La ausencia de información en nuestro país de una patología de tal importancia amerita su caracterización dentro de nuestra población así como evaluar el rendimiento de medidas de evaluación del riesgo, ya que no se cuenta con una herramienta definitiva que resuelva la incertidumbre diagnóstica y pronóstica, ni se cuenta con estudios que establezcan estos determinantes en Colombia y Latinoamérica (3-10).

El presente trabajo pretende determinar la factibilidad de un estudio de comparación de reglas de decisión clínica en síncope, poner a prueba la logística, mejorar la calidad de los procedimientos y recopilar información antes de que se lleve a cabo un estudio más amplio. Realizar un estudio piloto puede exponer los problemas en el diseño metodológico y procedimental y ser tratados antes de que el estudio se lleve a cabo en una investigación a mayor escala.

El objetivo primario es desarrollar un estudio piloto sobre la factibilidad de evaluación del rendimiento pronóstico de reglas de riesgo en síncope. Como objetivo secundario se propone evaluar el rendimiento pronóstico de las reglas de decisión clínica OESIL (11) (Osservatorio Epidemiologico sulla Síncope nel Lazio), EGSYS (12) (European Guidelines in Syncope Study), SFSR (13) (San Francisco Syncope Rule) y su aplicación con los Ottawa Electrocardiographic Criteria (SFSR+Ottawa) (14) en la predicción de mortalidad por cualquier causa y desenlaces cardiovasculares y no cardiovasculares mayores a siete y 30 días en pacientes adultos con diagnóstico de síncope en urgencias.

\section{Material y métodos}

El trabajo corresponde a un estudio observacional, analítico, prospectivo, de tipo longitudinal y de no intervención. Los criterios de inclusión fueron pacientes mayores de 18 años admitidos al servicio de urgencias del Hospital Fundación San Carlos con síncope de menos de 48 horas de evolución. Se contó con aprobación previa del comité de ética institucional para el desarrollo de la investigación.

Los pacientes fueron incluidos prospectivamente en forma consecutiva mediante muestreo por conveniencia. Se excluyeron pacientes con pérdida de la conciencia sugestiva de origen diferente al síncope, como la asociada a consumo de alcohol, hipoglucemia, sedantes o drogas ilícitas, trauma de cráneo previo a la pérdida de conciencia, antecedente de epilepsia conocida con un episodio presenciado o descrito por testigos de convulsión típico y posictal prolongado, déficit neurológico persistente sugestivo de ACV, reclutamiento previo en el estudio o que no firmaran el consentimiento.

El seguimiento se realizó vía telefónica a los siete y 30 días de la presentación del evento y mediante evaluación de historias clínicas de pacientes que se encontrasen aún hospitalizados.

Se aplicaron las reglas de predicción clínica de acuerdo con las variables presentadas en los artículos originales. En resumen estas variables incluyen para la SFSR (13): electrocardiograma anormal, disnea, hematocrito menor de $30 \%$, presión arterial sistólica $<90 \mathrm{mmHg}$ al ingreso a triage o historia de insuficiencia cardiaca congestiva.

Para la SFSR con los criterios electrocardiográficos de Otawa (14) se aplican las mismas variables de la SFSR pero se considera anormal el ECG sólo en presencia de: bloqueo auriculoventricular de segundo grado Mobitz tipo 2 o tercer grado, bloqueo completo de rama derecha o izquierda + bloqueo AV de primer grado, bloqueo completo de rama derecha + hemibloqueo anterosuperior o posteroinferior, cambios isquémicos nuevos, ritmo no sinusal, desviación del eje hacia la izquierda, anormalidades en el monitor cardiaco.

Para la regla OESIL (11): edad mayor a 65 años, enfermedad cardiovascular en la historia clínica, ausencia de pródromos sincopales, electrocardiograma anormal.

Para la regla EGSYS (12): palpitaciones precediendo al síncope, enfermedad cardiaca, electrocardiograma anormal, síncope durante esfuerzo, síncope en posición supina, factores precipitantes o predisponentes (sitios calurosos y 
abarrotados/ortostasia prolongada/miedo-dolor-emoción), pródromos autonómicos (náusea-vómito).

Las variables cualitativas fueron reportadas como porcentajes y las variables cuantitativas con medidas de tendencia central y de dispersión.

Para la evaluación de asociaciones entre las variables independientes categóricas con la variable dependiente "desenlace", se utilizó la prueba chi-cuadrado de Pearson (valores esperados $>5$ ) o la prueba exacta de Fisher (valores esperados $<=5$ ). La fuerza de asociación entre los factores se evaluó con el riesgo relativo (RR) y se determinó la asociación con el intervalo de confianza de $95 \%$.

El modelo utilizado en la explicación del "desenlace" ajustado por las variables de confusión fue la regresión logística binaria (la variable dependiente es binaria, es decir que sólo puede adquirir dos posibles valores (Sí-No, 0-1), las variables incluidas en el modelo fueron las significativas en el análisis bivariado, es decir, significancia con $\mathrm{p}<0.05$.

Para la comparación del rendimiento de las reglas de decisión clínica se realizaron curvas ROC para determinar el área bajo la curva para los desenlaces que fueron evaluados. Se determinó el rendimiento de EGSYS, OESIL, SFSR y SFSR+Ottawa en la predicción de mortalidad y/o desenlaces mayores predefinidos.

Se definieron como desenlaces mayores:

El desenlace muerte constatado mediante historia clínica, otros registros clínicos, o información obtenida por interrogatorio durante seguimiento telefónico de familiares o acudientes.

Infarto agudo al miocardio: se definió como cuadro clínico compatible por dolor, disnea o síncope y elevación de troponina significativa o cambios electrocardiográficos, con dicho diagnóstico al egreso como confirmación médica.

Arritmia: en registro ECG o monitor con relación de temporalidad al episodio sincopal o requiriendo tratamiento.

Embolismo pulmonar: determinado por angiotomografía de tórax, gammgrafía de ventilación, perfusión o angiografía pulmonar y al diagnóstico de egreso, recibiendo el paciente tratamiento para el mismo o confirmación por necropsia si se realizó.

ACV: diagnóstico al egreso, relacionado temporalmente con la admisión a la institución hospitalaria.

Hemorragia subaracnoidea: diagnóstico al egreso, relacionado temporalmente con la admisión a la institución hospitalaria.

Hemorragia significativa: cualquier episodio de síncope asociado con una fuente de sangrado que requiera transfusión.

Reingreso: cualquier paciente dado de alta de la institución hospitalaria después de un episodio sincopal y readmitido por los mismos síntomas o similares y que requiere una intervención aguda.

Intervención aguda: cualquier procedimiento requerido para tratar una condición relacionada con los síntomas sincopales: (inserción de marcapasos, cirugía valvular cardiaca, inserción de balón de contrapulsación, uso de vasopresores, cirugía para tratar aneurisma aórtico abdominal, ruptura esplénica, tratamiento endoscópico de varices esofágicas, inicio de terapia dialítica, reanimación cardiopulmonar, intervención coronaria percutánea, inserción de marcapasos o cardiodesfibrilador, cirugía valvular cardiaca, cirugía para tratar aneurisma aórtico abdominal, cirugía de revascularización miocárdica u otra cirugía cardiaca, inserción de balón de contrapulsación aortica, uso de medicación vasopresora endovenosa, uso de medicación antiarrítmica, traslado a unidad de cuidado crítico (Intermedio o intensivo), cirugía por estenosis carotídea, cirugía por ruptura esplénica, cirugía por embarazo ectópico roto u otra cirugía mayor.

Rabdomiolisis relacionada con el episodio sincopal.

Fractura(s) de columna secundarias a trauma debido al episodio sincopal.

Se consideraron también desenlaces los siguientes hallazgos entre los estudios realizados al paciente posteriores a la presentación del episodio sincopal: ecocardiograma con obstrucción mayor al flujo sanguíneo o estenosis valvular severa, evidencia clínica o electrocardiográfica de isquemia miocárdica, monitoreo o ECG con bradicardia sinusal (40 lpm), bloqueos sinoatriales repetitivos, pausas sinusales $>$ 3 segundos, Mobitz II o bloqueo AV avanzado (2:1, 3:1), bloqueo AV tercer grado, bloqueo alternante rama derecha o izquierda, disfunción marcapasos con pausas sinusales, taquiarritmia supraventricular paroxística, taquiarritmia ventricular. Holter-ECG con cualquier arritmia asociada a síncope o en ausencia de síntomas de pérdida de conciencia durante el registro la presencia de pausas ventriculares mayores a tres segundos al despertar, bloqueo AV Mobitz 2 segundo grado o avanzado, o tercer grado o taquiarritmia ventricular paroxística.

Estudio electrofisiológico con alguno de los siguientes hallazgos: bradicardia sinusal y tiempo corregido de recuperación del nodo sinusal mayor a $525 \mathrm{~ms}$, intervalo HV $>100$ ms, aparición de bloqueo AV infrahisiano de segundo o tercer grado durante marcapasos auricular, inducción de taquicardia ventricular monomórfica sostenida y síncope o taquiarritmia supraventricular hipotensora,

Prueba de esfuerzo con anormalidades ECG y presentación de síncope durante el ejercicio o inmediatamente posterior, desarrollo de bloqueo AV Mobitz 2 o de tercer grado durante ejercicio (incluso sin síncope).

Finalmente se determinó la sensibilidad, especificidad, VPP y VPN de las cuatro reglas de decisión para predicción de muerte y/o cualquier desenlace mayor a 7 y 30 días. Se diseñó un instrumento para recolección y registro de datos. Se empleó el programa SPSS versión 17.0 como herramienta para el análisis estadístico (SPSS, Inc., Chicago, IL).

\section{Resultados}

Se evaluaron pacientes con síncope durante el periodo de tiempo del 1 de julio de 2012 al 31 enero de 2013 que ingresaron al servicio de urgencias del Hospital Fundación 
San Carlos. Se recolectaron 44 casos a quienes mediante el instrumento de recolección de datos se les evaluaron 55 variables definidas a partir de estudios previos. A cada uno de los sujetos se le aplicó las reglas de decisión clínica OESIL, EGSYS, SFSR y la aplicación de esta última implementando los criterios electrocardiográficos de Ottawa (SFSR+Ottawa), realizándose seguimiento a los siete y 30 días para determinar la ocurrencia de desenlaces mayores y muerte.

Las características del síncope, el estado clínico al ingreso y comorbilidades de los sujetos incluidos en el estudio se muestran en la Tabla 1.

Tabla 1. Características del síncope, estado clínico al ingreso y comorbilidades.

\begin{tabular}{|c|c|c|c|c|c|}
\hline \multicolumn{2}{|c|}{ Descripcion } & Mínimo & Máximo & Media & $\begin{array}{c}\text { Desviación } \\
\text { estándar }\end{array}$ \\
\hline \multicolumn{2}{|l|}{ Edad } & 18 & 98 & 68.4 & 19.2 \\
\hline \multicolumn{2}{|c|}{ Hemoglobina } & 5.4 & 19.0 & 13.4 & 2.6 \\
\hline \multicolumn{2}{|c|}{ Hematocrito } & 15.0 & 56.3 & 40.3 & 7.6 \\
\hline \multicolumn{2}{|c|}{ Glucometría / glucemia } & 70 & 302 & 125 & 56.3 \\
\hline \multicolumn{2}{|c|}{ TA media } & 50 & 144.3 & 93.4 & 17.9 \\
\hline \multicolumn{2}{|c|}{ TA sistólica } & 70 & 210 & 128.4 & 27.7 \\
\hline \multicolumn{2}{|c|}{ TA diastólica } & 40 & 126 & 75.8 & 16.2 \\
\hline \multicolumn{2}{|c|}{ FC triage } & 45 & 124 & 79.9 & 18.5 \\
\hline & & & \multicolumn{2}{|l|}{$\mathbf{N}$} & Porcentaje (\%) \\
\hline \multirow[t]{2}{*}{ Sexo } & \multicolumn{2}{|c|}{ Mujeres } & 22 & \multicolumn{2}{|r|}{50.0} \\
\hline & \multicolumn{2}{|c|}{ Hombres } & 22 & & 50.0 \\
\hline Raza & & & 3 & & 6.8 \\
\hline & & & 41 & & 93.2 \\
\hline $\begin{array}{r}\text { Carac } \\
\text { Di }\end{array}$ & ínco & & 12 & & 27.3 \\
\hline & & & 4 & & 9.1 \\
\hline & oint & & 3 & & 6.8 \\
\hline & & & 13 & & 29.5 \\
\hline & & & 8 & & 18.2 \\
\hline & & & 2 & & 4.5 \\
\hline & & & 5 & & 11.4 \\
\hline & teric & & 6 & & 13.6 \\
\hline & & & 31 & & 70.5 \\
\hline & & & 3 & & 6.8 \\
\hline & & & 12 & & 27.3 \\
\hline & & & 17 & & 38.6 \\
\hline & & & 21 & & 47.7 \\
\hline & & & 1 & & 2.3 \\
\hline Antec & & & & & \\
\hline & Ictur & iaca & 19 & & 43.2 \\
\hline & & & 10 & & 22.7 \\
\hline & ial $p$ & & 3 & & 6.8 \\
\hline $\mathrm{Ar}$ & ular & & 2 & & 4.5 \\
\hline & na a & previo & 4 & & 9.1 \\
\hline & & & 15 & & 34.1 \\
\hline & iliar & súbita & 1 & & 2.3 \\
\hline & setab & dores & 9 & & 20.5 \\
\hline $\mathrm{Fa}$ & calci & nistas & 11 & & 25.0 \\
\hline $\mathrm{Fa}$ & antia & & 1 & & 2.3 \\
\hline & $\mathrm{EC}$ & A II & 20 & & 45.5 \\
\hline
\end{tabular}

Con respecto a los desenlaces evaluados a los siete y 30 días la frecuencia de presentación de los mismos se muestra en la Tabla 2.

El de las variables independientes categóricas $v s$ la variable dependiente desenlace mostró diferencia estadística significativa sugiriendo que son factores de riesgo para presentar muerte o desenlace mayor fueron las siguientes: desviación del eje (p: 0.031 OR: 9), electrocardiograma anormal (p: 0.003 OR: 9.58) e hipertrofia ventricular (p: 0.038 OR: 1.85$)$.

Otras variables que mostraron diferencia estadísticamente significativa y sugieren ser predictores de bajo riesgo para muerte o desenlace mayor fueron las siguientes: pródromos (p: 0.043 y OR 0.17 ), náuseas/vómito (p: 0.036 y OR 0.26 ) y visión borrosa (p: 0.045 y OR 0.23 ).

Se realizó regresión logística binaria con las variables que fueron significativas en el análisis bivariado y la variable troponina anormal que tuvo significancia estadística dentro del modelo. Ingresaron las variables EKG anormal y troponina anormal que explican el $45.7 \%$ del evento muerte y desenlace mayor. Las variables desviación del eje e hipertrofia fueron excluidas porque mostraron colinealidad entre sí y con la variable EKG anormal.

El análisis ROC de cada uno de los puntajes pronósticos para su desenlace original, sugiere que la regla de decisión clínica SFSR + Ottawa a siete días tiene adecuada capacidad predictiva con área bajo la curva $(\mathrm{AUC})>0.75$ para la

Tabla 2. Frecuencia de desenlaces presentados a siete y 30 días.

\begin{tabular}{|c|c|c|c|c|}
\hline \multirow[t]{2}{*}{ Desenlaces } & \multicolumn{2}{|c|}{ A 7 días } & \multicolumn{2}{|c|}{ A 30 días } \\
\hline & $\mathbf{N}$ & $\%$ & $\mathbf{N}$ & $\%$ \\
\hline Muerte por cualquier causa & & & 4 & 9 \\
\hline IAM & 2 & 4.5 & 1 & 2.3 \\
\hline Arritmias & 11 & 25 & 1 & 2.3 \\
\hline Ritmo no sinusal & 10 & 22.7 & & \\
\hline Pausas ventriculares & 1 & 2.3 & & \\
\hline Tromboembolismo pulmonar & 1 & 2.3 & & \\
\hline Evento cerebrovascular & 1 & 2.3 & & \\
\hline Hemorragia significativa & 4 & 9 & & \\
\hline Sepsis severa / choque séptico & 2 & 4.5 & & \\
\hline Intervenciones agudas & 5 & 11.3 & & \\
\hline Intervención coronaria percutánea & 3 & 6.8 & 1 & 2.3 \\
\hline Marcapasos & 2 & 4.5 & & \\
\hline Vasopresores & 1 & 2.3 & & \\
\hline Antiarrítmicos & 1 & 2.3 & & \\
\hline Unidad cuidado intensivo & 7 & 15.9 & 1 & 2.3 \\
\hline Cirugía mayor & 1 & 2.3 & & \\
\hline $\begin{array}{l}\text { Tratamiento endoscópico: } \\
\text { varices esofágicas }\end{array}$ & 1 & 2.3 & & \\
\hline Diálisis & 1 & 2.3 & & \\
\hline Eco obstrucción flujo & 2 & 4.5 & & \\
\hline Ecg isquemia & 7 & 15.9 & & \\
\hline Monitoreo/ECG pausas sinusales & 1 & 2.3 & & \\
\hline Monitoreo/ecg Mobitz II / $(2: 1 ; 3: 1)$ & 1 & 2.3 & & \\
\hline Monitoreo/ECG TSVP & 1 & 2.3 & & \\
\hline Holter alterado sin síntomas & 1 & 2.3 & & \\
\hline Reingreso & 2 & 4.5 & & \\
\hline
\end{tabular}


variable muerte y/o desenlaces mayores, mostrando AUC de 0.76 (IC 95\% 0.618-0.903) y 30 días con AUC de 0.73 (IC 95\% 0.584-0.881)

Es de aclarar que para OESIL y EGSYS no se calculó el AUC ni sensibilidad, especificidad, VPP y VPN a siete días por qué no se presentó el desenlace predefinido mortalidad para estos puntajes en los sujetos en seguimiento durante este periodo de tiempo. Las muertes fueron posteriores al séptimo día de seguimiento. Su valor a 30 días se muestra en la Tabla 3. En la Tabla 4 y las Figuras 1-3 se puede apreciar la AUC para cada escala utilizada.

Adicionalmente se determinó la sensibilidad, especificidad, valor predictivo positivo y valor predictivo negativo de las reglas de decisión en la predicción de muerte y/o desenlaces mayores aplicado a siete y 30 días, como se muestra en la Tabla 5.

Los cuatro casos de mortalidad se describen a continuación: uno de los casos ingresó con un síncope considerado como situacional postusivo, diagnosticándose durante su estancia una masa pulmonar con diagnóstico confirmado histológicamente de carcinoma de pulmón avanzado y derrame pleural asociado. Las cuatro reglas de decisión fueron positivas.

El segundo paciente ingresó con hemorragia de vías digestivas y síncope por hipotensión ortostática. Las cua- tro reglas de decisión fueron negativas no prediciendo el desenlace. La anemización de acuerdo con los valores de la regla de San Francisco fue posterior al ingreso y en este caso la tensión arterial de ingreso fue de 90/60 estando en el límite del criterio de positividad. Por su condición clínica fue hospitalizado al ingreso y manejado en unidad de cuidado crítico donde murió.

El tercer caso se clasificó clínicamente como síncope neuromediado en un sujeto de 81 años que tenía como hallazgo electrocardiográfico un bloqueo auriculoventricular de primer grado, presentándose al seguimiento telefónico muerte domiciliaria sin causa aclarada. Todas las reglas de decisión exceptuando EGSYS resultaron positivas.

El último caso se clasificó clínicamente como secundario a hipotensión ortostática en un sujeto anciano con deterioro cognitivo y dependencia funcional presentándose al seguimiento telefónico muerte domiciliaria. Las reglas de decisión clínica fueron positivas excepto por EGSYS.

\section{Discusión}

Este estudio se realizó como piloto para evaluación de factibilidad y desempeño del instrumento de recolección de datos, proceso de inclusión y evaluación de método de estudio y factibilidad de reclutamiento de muestra, precediendo la realización de un estudio multicéntrico en Colombia para

Tabla 3. AUC Reglas de decisión para desenlace original.

\begin{tabular}{|c|c|c|c|c|c|}
\hline \multicolumn{6}{|c|}{ AUC Reglas de decisión } \\
\hline \multirow{2}{*}{ Regla de decisión } & \multirow{2}{*}{ Área } & \multirow{2}{*}{ Error estándar } & \multirow{2}{*}{$p$} & \multicolumn{2}{|c|}{ Intervalo de confianza $\mathbf{9 5 \%}$} \\
\hline & & & & Límite inferior & Límite superior \\
\hline SFSR siete días & 0.656 & 0.082 & 0.077 & 0.495 & 0.817 \\
\hline SFSR 30 días & 0.658 & 0.083 & 0.077 & 0.496 & 0.820 \\
\hline SFSR+Ottawa siete días & 0.76 & 0.073 & 0.003 & 0.618 & 0.903 \\
\hline SFSR+Ottawa 30 días & 0.733 & 0.076 & 0.009 & 0.584 & 0.881 \\
\hline OESIL 30 días & 0.519 & 0.135 & 0.903 & 0.253 & 0.784 \\
\hline EGSYS 30 días & 0.256 & 0.117 & 0.111 & 0.026 & 0.487 \\
\hline
\end{tabular}

Tabla 4. Rendimiento pronostico reglas de decisión para muerte y/o desenlaces mayores.

\begin{tabular}{|c|c|c|c|c|}
\hline & \multirow{2}{*}{$\begin{array}{c}\text { Sensibilidad } \\
(\%)\end{array}$} & \multirow{2}{*}{$\begin{array}{c}\text { Especificidad } \\
(\%)\end{array}$} & \multicolumn{2}{|c|}{ Valor predictivo (\%) } \\
\hline & & & Positivo & Negativo \\
\hline $\begin{array}{l}\text { SFSR } \\
\text { A siete días } \\
\text { A } 30 \text { días }\end{array}$ & $\begin{array}{l}79 \\
81\end{array}$ & $\begin{array}{l}35 \\
39\end{array}$ & $\begin{array}{l}59 \\
66\end{array}$ & $\begin{array}{l}58 \\
58\end{array}$ \\
\hline $\begin{array}{l}\text { SFSR+OTTAWA } \\
\text { A siete días } \\
\text { A } 30 \text { días }\end{array}$ & $\begin{array}{l}86 \\
84\end{array}$ & $\begin{array}{l}45 \\
47\end{array}$ & $\begin{array}{l}61 \\
68\end{array}$ & $\begin{array}{l}77 \\
69\end{array}$ \\
\hline $\begin{array}{l}\text { OESIL } \\
\text { A siete días } \\
\text { A } 30 \text { días }\end{array}$ & $\begin{array}{l}65 \\
65\end{array}$ & $\begin{array}{l}52 \\
56\end{array}$ & $\begin{array}{l}60 \\
68\end{array}$ & $\begin{array}{l}58 \\
53\end{array}$ \\
\hline $\begin{array}{l}\text { EGSYS } \\
\text { A siete días } \\
\text { A } 30 \text { días }\end{array}$ & $\begin{array}{l}61 \\
58\end{array}$ & $\begin{array}{l}62 \\
61\end{array}$ & $\begin{array}{l}64 \\
68\end{array}$ & $\begin{array}{l}59 \\
50\end{array}$ \\
\hline
\end{tabular}

Tabla 5. Rendimiento pronostico reglas de decisión OESIL y EGSYS para desenlace original muerte.

\begin{tabular}{|l|c|c|c|c|}
\hline & \multirow{2}{*}{$\begin{array}{c}\text { Sensibilidad } \\
(\%)\end{array}$} & $\begin{array}{c}\text { Especificidad } \\
(\%)\end{array}$ & \multicolumn{2}{|c|}{ Valor predictivo (\%) } \\
\cline { 4 - 5 } & & & Positivo & Negativo \\
\hline $\begin{array}{l}\text { OESIL } \\
\text { A 30 días }\end{array}$ & $50 \%$ & $43 \%$ & $8 \%$ & $89 \%$ \\
\hline $\begin{array}{l}\text { EGSYS } \\
\text { A 30 días }\end{array}$ & $25 \%$ & $48 \%$ & $5 \%$ & $86 \%$ \\
\hline
\end{tabular}




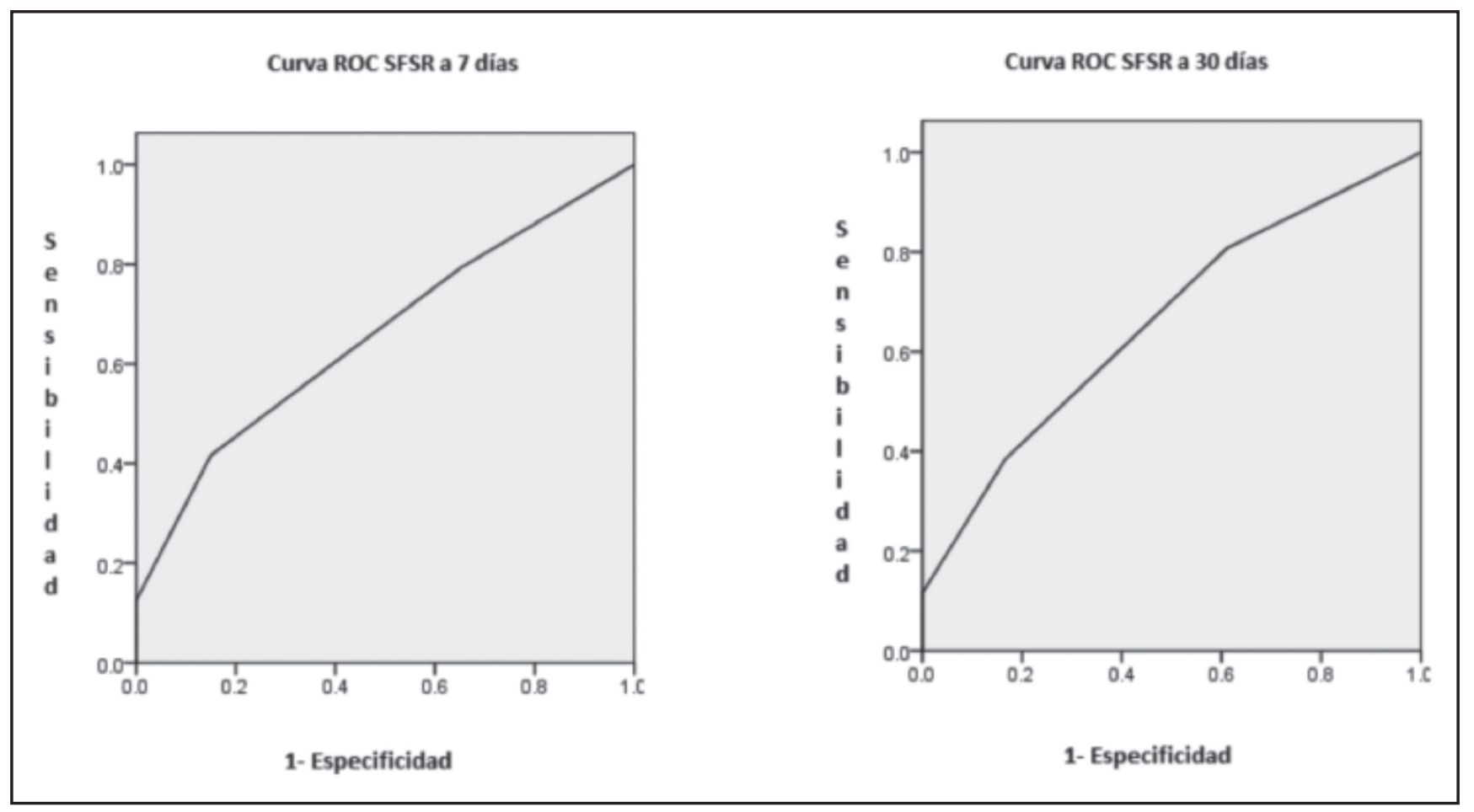

Figura 1. Curvas ROC regla de decisión SFSR.

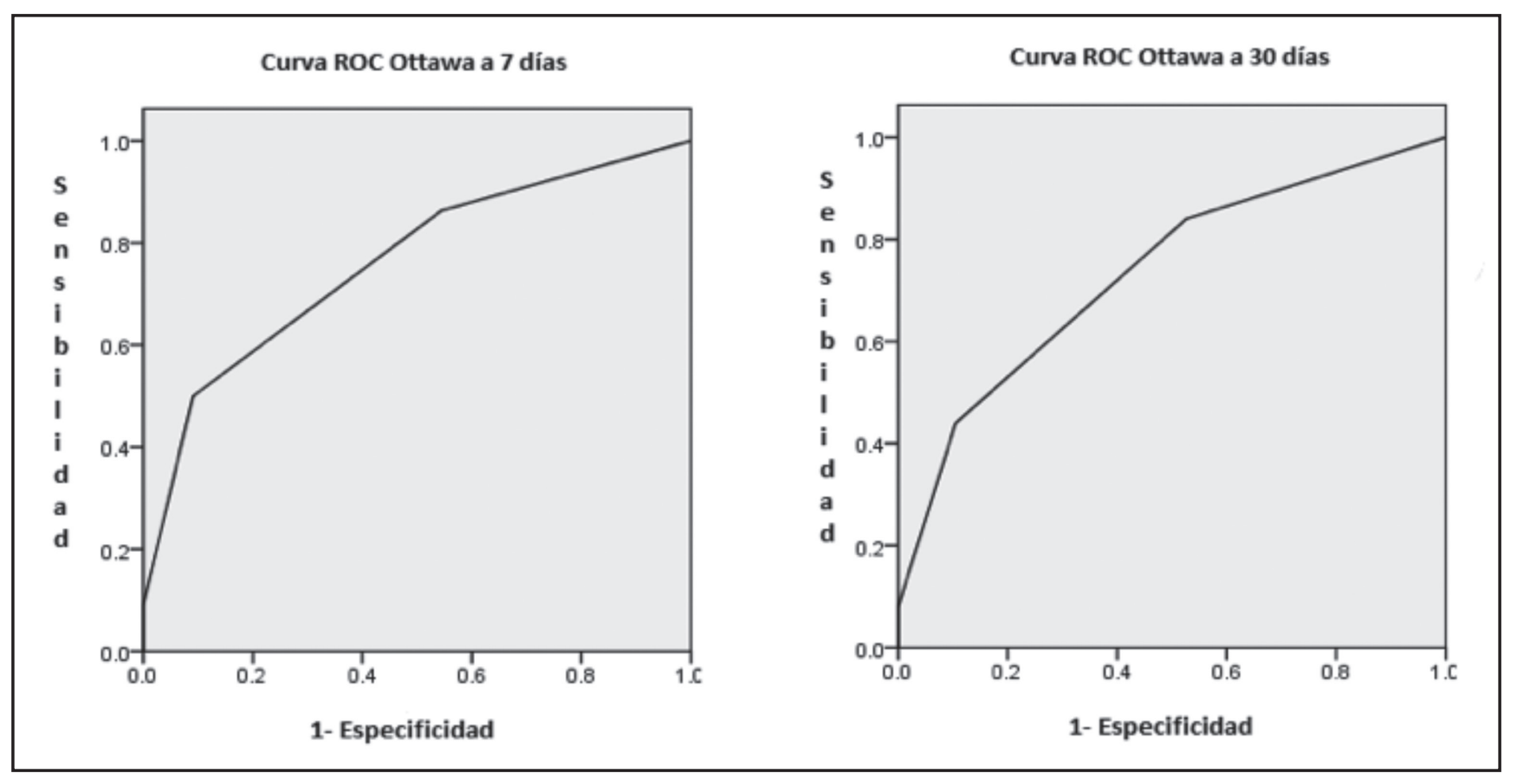

Figura 2. Curvas ROC regla de decisión SFSR + Ottawa.

la evaluación de reglas de riesgo en síncope en pacientes que consultan a urgencias. El modelo de estudio piloto ha sido utilizado dentro de la investigación del síncope optimizando los recursos de investigación al corregir fallas en instrumentos de recolección, inclusión de sujetos de estudio como en el caso de la derivación de la regla ROSE (15).
Las características de la población en estudio difieren de otros estudios de síncope. La totalidad de los pacientes incluidos fueron hospitalizados, aunque se presentaron egresos hacia las 24 horas. Esto denota posiblemente la mayor sensibilidad de los médicos tratantes a la relación de síncope con mortalidad ulterior, pero establece claramente la nece- 


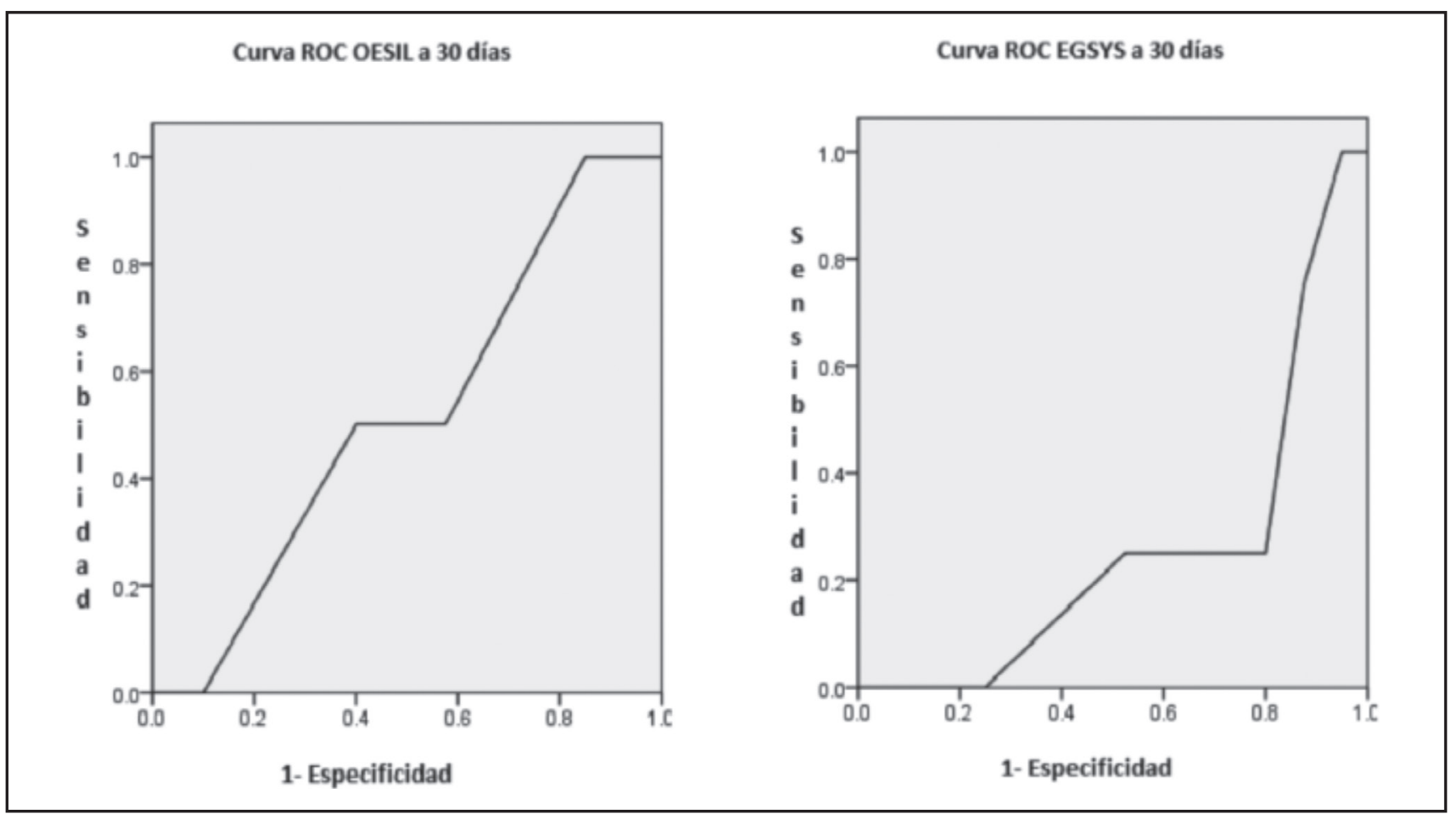

Figura 3. Curvas ROC reglas de decisión OESIL y EGSYS.

sidad de establecer reglas de decisión clínica para disminuir los costos de hospitalizar $100 \%$ de pacientes con síncope.

La conducta adoptada por los médicos tratantes fue independiente de las reglas de decisión clínica, por ello surgen diferencias metodológicas principalmente con OESIL y EGSYS, pues no se contó con supervisión del servicio de cardiología o evaluación de adherencia a guías de manejo por el carácter de no intervención. Sin embargo, se relaciona más con la realidad de la mayoría de instituciones de salud de nuestro país, que no tienen un servicio de cardiología disponible permanentemente para pacientes que llegan a urgencias.

En esta cohorte la edad promedio (68.4 años) fue mayor a la de los estudios de validación de las reglas (62 años para San Francisco, 58.5 para Ottawa, 57 años para OESIL y 61 para EGSYS.

Nuestro estudio muestra una alta tasa de prevalencia de arritmias $(25 \%)$ que también es mayor a la de estudios previos. Esto sugiere una población más enferma (que se correlaciona con la mayor edad promedio) pero que también tiene la limitante de la definición de arritmia al igual que la definición de ECG anormal. Desde lo amplio del concepto de ECG anormal en la regla de síncope de San Francisco, hasta una definición más estricta de los criterios de ECG anormal de Ottawa u OESIL. Adicionalmente se ha atribuido la variabilidad en el rendimiento pronóstico obtenido en la validación de estos puntajes a no incluir todos los ECG o monitoreos electrocardiográficos realizados a los sujetos de estudio, sino sólo el primer registro para el caso de SFSR dentro del análisis, reflejándose en un rendimiento variable entre estudios de comparación para sensibilidad y especificidad. Para el presente estudio se ha reproducido la aplicación de los criterios señalados en cada regla de decisión clínica para acercarse al mayor rendimiento obtenido en los estudios de derivación; aunque estos algoritmos muchas veces se consideran aplicables después de no lograr aclarar mediante la aproximación médica inicial y el juicio clínico, la decisión del escenario de diagnóstico y manejo de un paciente.

El ejercicio de comparación de rendimiento pronóstico se desarrolló con desenlaces a 7 y 30 días a pesar de que para las reglas de decisión OESIL y EGSYS se derivó la regla con base en desenlaces a 12 y 21-24 meses respectivamente. La intención fue aproximarse a su utilidad en el pronóstico a corto plazo, al encontrarse poca información de estudios que interrogaran esa pregunta, sin contarse con evaluación de adherencia a guías clínicas de manejo o supervisión por cardiología. Adicionalmente se encuentra en este estudio piloto el hecho de usar EGSYS como predictor de mortalidad y no como predictor diagnóstico de síncope cardiogénico (desenlace en el estudio original), con el supuesto de mayor riesgo de mortalidad por esta etiología de síncope, aunque no se encontró peso de este tipo de síncope en los desenlaces fatales. Se respetó la definición histórica de desenlaces de las reglas previas como en el caso de infarto agudo al miocardio en lugar de usarse la nueva definición (16) para emplear la usada en dicho estudio, así como la inclusión en el instrumento de recolección de los desenlaces tenidos en cuenta en cada una de las reglas analizadas. Adicionalmente 
no se encontró una validación prospectiva de aplicación de la regla SFSR modificada mediante la aplicación de los criterios de Ottawa, lo cual consideramos una primera aproximación al rendimiento pronóstico de Ottawa con miras a futuros estudios.

Con respecto al desempeño de la regla de decisión SFSR, en validaciones posteriores al estudio inicial se encuentran valores más bajos de sensibilidad y especificidad en la mayoría de estudios, aunque con variación en el tipo de estudio y edad de los individuos, sin lograr reproducir el rendimiento del estudio original en gran parte de ellos (17). Posteriores publicaciones de los autores de SFSR indican como causa de esta heterogeneidad la aplicación variable de la regla, el momento de aplicación de la misma o las diversas interpretaciones del ECG como anormal, no lográndose una satisfactoria validez externa (17-21).

Un metaanálisis incluyó doce estudios con un $\mathrm{n}=5316$ y desenlaces mayores del 11\%, determinó una sensibilidad de $87 \%$ y especificidad de $52 \%$ con una alta heterogeneidad de los estudios en lo referente al intervalo para sensibilidad (55-98\%) que se atribuyó por los autores a la interpretación de la variable ECG anormal y por la aplicación inconsistente de la definición de arritmias significativas. Como aspecto por resaltar se describe que la probabilidad de un desenlace mayor es menor de $2 \%$ cuando se aplica la regla de decisión después de no lograr identificar la causa del síncope mediante la evaluación médica inicial (22). En nuestro estudio la sensibilidad fue de 79 y $81 \%$ a 7 y 30 días, con baja especificidad de 35 y $39 \%$ respectivamente.

En este estudio piloto se encontró, de acuerdo con las características operativas predefinidas de comparación, un mejor rendimiento pronóstico para SFSR+Ottawa seguido de SFSR según el área bajo la curva obtenida y sensibilidad con significancia estadística, aunque con una baja especificidad para lo que se considera ideal en este tipo de instrumentos de medición clínica en general, que sacrifican especificidad a expensas de una sensibilidad cercana al $100 \%$.

No es posible descartar la utilidad de OESIL y EGSYS por limitantes como el objetivo primario y tamaño de este estudio y la salvedad hecha con los tiempos de medición de desenlace de estas reglas, concebidas para pronóstico a largo plazo.

Cabe señalar que para la aplicación de los criterios electrocardiográficos de Ottawa y en general dentro del estudio, no se realizó evaluación de concordancia interobservador en el análisis de electrocardiograma el cual en la mayoría de casos era interpretado por el médico internista, aunque en los casos de valoración por cardiología se consideraba el diagnóstico de este servicio. Esta forma de aplicación dentro del contexto de no intervención se aproxima más a la aplicación de las reglas en escenarios reales de práctica clínica.

Esta cohorte tuvo una alta mortalidad (10\%) comparada con San Francisco (0.7\%), Ottawa (0.4\%), aunque similar a la de estudios como OESIL (11.5\%) y EGSYS (9\%). Sin embargo, el alto número de hospitalizaciones y de desen- laces mayores si bien pudiera explicarse por el tamaño de muestra sugiere una mayor gravedad en el estado de salud de los pacientes incluidos. En este sentido se encuentra, por las características de la población en cuanto a sus antecedentes, un alto porcentaje de pacientes con medicación de patología cardiovascular (uso de IECA/ARAII $45 \%$ vs. $16.4 \%$ SFSR), mayor enfermedad cardiaca estructural de acuerdo con la definición EGSYS (43.2\% vs. 30\% EGSYS; $23.8 \%$ OESIL). Hubo menor antecedente de enfermedad coronaria (11.36\% vs. 24.1\% SFSR; $19.6 \%$ Ottawa). Si bien el desenlace isquemia e IAM fue importante en este estudio, el peso de mortalidad fue dado por hemorragia digestiva, patología neoplásica y causas no determinadas.

En cuanto al desenlace muerte para la población evaluada surgen dos limitantes: la ausencia de pruebas adicionales para confirmar la causa del síncope como mesa basculante o estudio electrofisiológico; aunque en las muertes presentadas probablemente no existía indicación y el no contar con diagnóstico posmortem mediante necropsia. En el caso de la aplicación de la regla EGSYS, ésta permite orientar hacia la etiología del síncope como cardiogénico, pero el hecho de que éste no sea cardiogénico probablemente no lo tipifica como benigno o de bajo riesgo por sí solo.

\section{Limitaciones}

Al tratarse de un estudio piloto existen limitantes en la interpretación de los desenlaces, en especial en la comparación del rendimiento pronóstico de las reglas de decisión aplicadas por el tamaño de la muestra. La totalidad de pacientes fueron hospitalizados por lo que no podría aplicarse sus resultados a pacientes con síncope que no se hospitalicen, una de las decisiones que las reglas en general pretenden definir. Por tanto, deberá optimizarse el proceso de inclusión de los pacientes para lograr la recolección de sujetos de estudio con aparente bajo riesgo, objetivo que también puede facilitarse al desarrollarlo de manera multicéntrica.

\section{Conclusiones}

Este trabajo constituye la primera evaluación prospectiva de reglas de síncope en Colombia. Como piloto demostró que es factible realizar un estudio de comparación de rendimiento pronóstico. Sin embargo, se requiere incluir múltiples centros de atención con diferente nivel de complejidad y tiempo de respuesta en la realización de procedimientos diagnósticos y terapéuticos para acercarse hacia su implicación en los desenlaces. Deberá mejorarse el proceso de reclutamiento de los sujetos de estudio para que se incluyan casos tanto hospitalizados como de manejo ambulatorio después de su evaluación en los servicios de urgencias, logrando así extrapolar los resultados a diferentes escenarios. El presente estudio logró implementar satisfactoriamente el instrumento de recolección de datos y el proceso metodológico con miras al desarrollo de un futuro estudio multicéntrico.

Como no existe, y posiblemente no existirá, una regla de decisión clínica con sensibilidad y especificidad del 100\% 
(que se constituya en estándar de oro) contra la cual se comparen sus características operativas, este trabajo sugiere un buen desempeño de SFSR si se definen más estrictamente sus criterios de anormalidad electrocardiográfica (criterios de Ottawa). Por otra parte se plantea el interrogante del real desempeño de reglas de decisión clínica en la predicción de desenlaces a largo plazo cuando son empleadas en escenarios de corto plazo, que necesariamente requieren un estudio cuyo objetivo primario sea determinar sus características operativas y utilidad clínica con diferentes periodos de seguimiento.

Es manifiesta la necesidad de desarrollar investigación en síncope para determinar la mortalidad en nuestro país dado los resultados del presente trabajo en relación con el resto del mundo mediante la caracterización de nuestra población, la posible influencia de variables socioeconómicas y aquellas propias de nuestro sistema de salud así como la posibilidad de derivar y validar una regla de decisión construida en nuestro medio de ejercicio clínico.

\section{Fuentes de financiación}

Recursos propios

\section{Posibles conflictos de interés}

Ninguno. Arnold Méndez Toro no ha recibido ningún tipo de financiación para la realización de este trabajo por parte de compañías farmacéuticas o patrocinadores relacionados ni declara actividades previas o presentes con patrocinio de compañías farmacéuticas que pudiesen generar conflictos de interés. Ingrid Tatiana Rojas Ruiz no ha recibido ningún tipo de financiación para la realización de este trabajo por parte de compañías farmacéuticas ni otros patrocinadores. Tampoco declara actividades previas o presentes bajo patrocinio de compañías farmacéuticas que pudiesen generar conflictos de interés. Óscar Ernesto Amarís Peña no ha tenido ningún tipo de financiación para la realización de este trabajo, ni declara al momento actividades con patrocinio de compañías farmacéuticas que pudiesen generar conflictos de interés. Guillermo Mora Pabón no ha tenido ningún tipo de financiación para la realización de este trabajo. Por otra parte ha recibido honorarios del laboratorio Boehringer Ingelheim por presentación de conferencias relacionadas con anticoagulación

\section{Referencias}

1. Benditt DG. Adkisson WO. Approach to the patient with syncope: Venues, presentations, diagnoses. Cardiol Clin 2013; 31: 9-25.

2. Sheldon R. Syncope Diagnostic Scores. Prog Cardiovas Dis. 2013; 55: 390-395.

3. Asensio E, Orea A, Castillo L, Fraga JM, Colín E, Prieto JA. Síncope en el anciano: Hallazgos de pruebas complementarias en un Centro Hospitalario Universitario. Arch Cardiol Mex. 2009; 79(3): 201-206.
4. Consuegra MI. Muñoz JF. Síncope. Acta Neurol Colomb. 2004; 20: 72-76.

5. Iglézias JC. Lourenção Jr. A. Octagenários: resultados de 3659 necropsias (12 anos de observação). Rev. Bras. Gir. Gardiovasc. 1993; 8(1): 39-43.

6. Civera RG. Máñez RS. Granell RR. Rendimiento diagnóstico de un protocolo de estudio del síncope de causa no aparente. Rev Esp Cardiol 2001; 54: 425-430.

7. Pacheco A, Burusco S, Senosiáin MV. Prevalencia de procesos y patologías atendidos por los servicios de emergencia médica extrahospitalaria en España. An. Sist. Sanit. Navar. 2010; 33 (1): 37-46.

8. Mohaweche R, Vidal IS, Pulido M. Utilidad de la historia clínica en la evaluación del paciente con Síncope. Archivos Venezolanos de Farmacología y Terapéutica. 2003; 22 (2): 142-152.

9. Arce A. Cantero EM. Análisis de supervivencia de pacientes que consultan por síncope. valor de las escalas de riesgo. Rev Esp Cardiol. 2012; 65 (3): 363.

10.Cárdenas M. Vallejo M. Prevalencia de síncope en una muestra de mujeres mexicanas residentes de la ciudad de México. Arch Cardiol Mex 2009; 79(3): 197-200.

11. Colivicchi F, Ammirati F, Melina D, Guido V, Imperoli G, Santini M. OESIL (Osservatorio Epidemiologico sulla Síncope nel Lazio) Study Investigators. Development and prospective validation of risk stratification system for patients with syncope in the emergency department: the OESIL risk score. Eur Heart J. 2003; 24(9): 811-819.

12. Del Rosso A, Ungar A, Maggi R, Giada F, Petix NR, De Santo T, et al. Clinical predictors of cardiac syncope at initial evaluation in patients referred urgently to a general hospital: the EGSYS score. Heart 2008; 94(12): 1620-1626.

13. Quinn JV. Stiell IG, McDermott DA, Sellers KL, Kohn MA, Wells GA. Derivation of the San Francisco Syncope Rule to predict patients with short-term serious outcomes. Ann Emerg Med. 2004; 43(2): 224-32.

14. Thiruganasambandamoorthy V, Hess EP, Turko E. Defining abnormal electrocardiography in adult emergency department syncope patients: the Ottawa Electrocardiographic Criteria. CJEM 2012; 14(4): 248-58.

15. Reed M, Newby D. The Risk stratification Of Syncope in the Emergency department (ROSE) pilot study: a comparison of existing syncope guidelines. Emerg Med J. 2007; 24(4): 270-5.

16. Thygesen K, Alpert J, Jaffe A, et al. Writing Group on behalf of the Joint ESC/ ACCF/AHA/WHF Task Force for the Universal Definition of Myocardial Infarction. Third Universal Definition of Myocardial Infarction. Circulation. 2012; 126(16): 2020-2035

17. Miller C, Hoekstra J. Prospective validation of the San Francisco syncope rule: will it change practice? Ann Emerg Med 2006; 47: 455-6.

18. Sun BC, Mangione CM, Merchant G, Weiss T, Shlamovitz GZ, Zargaraff G, et al. External validation of the San Francisco syncope rule. Ann Emerg Med. 2007; 49(4): 420-7.

19. Cosgriff T, Kelly A, Kerr D. External validation of the San Francisco Syncope rule in the Australian context. Can J Emerg Med. 2007; 9(3): 157-61.

20. Birnbaum A, Esses D, Bijur P, Wollowitz A, Gallagher EJ, et al. Failure to validate the San Francisco Syncope rule in an independent emergency department population. Ann Emerg Med. 2008; 52(2): 151-9.

21. Schladenhaufen R, Feilinger S, Pollack M, Benenson R, Kusmiesz AL, et al. Application of San Francisco syncope rule in elderly ED patients. Am J Emerg Med. 2008; 26(7): 773-8.

22. Saccilotto RT, Nickel CH, Buvher HC. San Francisco Syncope Rule to predict short-term serious outcomes: a systematic review. CMAJ. 2011; 183(15): E111626. 